\title{
Os benefícios da extubação paliativa na qualidade de morte
}

The benefits of palliative extubation on the quality of death

Los beneficios de la extubación paliativa en la calidad de muerte

Recebido: 01/10/2018

Aprovado: 09/01/2019

Publicado: 15/05/2020
Mariana Fernandes Peixoto ${ }^{1}$

Fabio Bruno Silva Nascimento ${ }^{2}$

Beatriz Paschoini Andrade Silva ${ }^{3}$

Daniela Santana Polati Silveira ${ }^{4}$

0 estudo tem como objetivo apresentar revisão acerca do processo da extubação paliativa assim como seus benefícios na qualidade de morte. Considerou-se o período de 2012 a meados de 2017 e utilizou-se documentos científicos obtidos nas plataformas de dados, SciELO, PubMed, Embase, Cochrane. A busca foi realizada utilizando os descritores Extubação, Cuidados Paliativos e Comunicação em inglês e português. Para o estudo, selecionou-se 19 artigos, dos quais 33,33\% apresentaram o cuidado paliativo associado à qualidade de morte, 38,88\% apresentaram os cuidados paliativos inseridos na unidade de terapia intensiva, $27,77 \%$ realizaram a extubação paliativa para uma melhor qualidade de morte. Nos artigos selecionados observou-se a importância da equipe multidisciplinar bem preparada, assim como uma boa comunicação com a família e o paciente, bem como o desfecho de uma extubação paliativa na qualidade de morte.

Descritores: Comunicação; Cuidados paliativos; Extubação.

This study aims to present a review of the palliative extubation process and its benefits in the quality of death. It considered the period from 2012 to mid-2017 and used scientific documents from the databanks, SciELO, PubMed, Embase, and Cochrane. The search was carried out using the descriptors Extubation, Palliative Care, and Communication, in English and Portuguese. 19 articles were selected, from which $33.33 \%$ discussed palliative care associated with the quality of death, $38.88 \%$ discussed palliative care in the intensive care unit, $27.77 \%$ performed palliative extubation for a better quality of death. In the selected articles, the importance of a well-prepared multidisciplinary team was observed, as were communication with the family and the patient, and the outcome of a palliative extubation in the quality of death.

Descriptors: Communication; Palliative care; Airway extubation.

El estudio tiene como objetivo presentar revisión acerca del proceso de la extubación paliativa así como sus beneficios en la calidad de muerte. Se consideró el periodo de 2012 hasta mediados de 2017 y se utilizaron documentos científicos obtenidos en las plataformas de datos SciELO, PubMed, Embase, Cochrane. La búsqueda fue realizada utilizando los descriptores Extubación, Cuidados Paliativos y Comunicación en inglés y portugués. Para el estudio, se seleccionaron 19 artículos, de los cuales 33,33\% presentaron el cuidado paliativo asociado a la calidad de muerte, $38,88 \%$ presentaron los cuidados paliativos insertos en la unidad de terapia intensiva, $27,77 \%$ realizaron la extubación paliativa para una mejor calidad de muerte. En los artículos seleccionados se observó la importancia del equipo interdisciplinario bien preparado, así como una buena comunicación con la familia y el paciente, así como el desenlace de una extubación paliativa en la calidad de muerte.

Descriptores: Comunicación; Cuidados paliativos; Extubación traqueal.

1. Fisioterapeuta. Especialista em Fisioterapia Oncológica e Hospitalar. ORCID: 0000-0001-6629-5479 E-mail: mari_fernandes31@hotmail.com

2. Fisioterapeuta. ORCID: 0000-0001-9635-4230 E-mail: fabiobruno12.01@gmail.com

3. Fisioterapeuta. ORCID: 0000-0001-5952-5328 E-mail: beatriz_p.a.s@hotmail.com

4. Fisioterapeuta. Doutoranda em Reabilitação e Desempenho Funcional pela Faculdade de Medicina de Ribeirão Preto, Universidade de São Paulo. Docente do Curso de Fisioterapia na Universidade de Franca, SP, Brasil. ORCID: 0000-0001-7352-8445 E-mail: danisantana.fisio@hotmail.com 


\section{INTRODUÇÃO}

$\mathbf{E}$

ventos que levam a uma admissão na unidade de terapia intensiva (UTI) podem levar a uma piora dos quadros de sinais e sintomas de uma doença crônica, devido a complicações e intercorrências. A equipe multidisciplinar da UTI deve se atentar em realizar continuamente a evolução clinica desses pacientes, o que incluirá os cuidados paliativos quando o tratamento curativo não mais oferecer benefícios.

Em algumas situações, a morte é inevitável, e prorroga os altos custos psicológicos, sociais e financeiros não somente da equipe envolvida, mas também de pacientes e seus familiares. Em muitos casos, o tratamento adicional não atinge a proposta de tratamento do paciente, o que vem se tornando cada vez mais comum nas UTI 1,2.

As discussões acerca do cuidado paliativo em UTI ainda é recente no Brasil, por existir um despreparo para situações em que o paciente entra em cuidados paliativos, porém, questões assim estão presentes diariamente na rotina hospitalar. De acordo com a Organização Mundial de Saúde (OMS), 57,89\% das pessoas que morrem necessitam de cuidados paliativos na sua terminalidade, e estima-se que, em 2030, 15 milhões de novos casos oncológicos ao ano necessitarão assistência paliativa. O Brasil lida com um milhão de óbitos por ano e cerca de $70 \%$ dessas mortes ocorrem em hospitais, a grande maioria em unidades de terapia intensiva ${ }^{3}$. Apenas $14 \%$ dos pacientes que necessitam de cuidados paliativos em todo o mundo recebem esse tipo de atenção ${ }^{4}$.

0 cuidado paliativo é de extrema importância, independente de a condição médica ser aguda ou crônica, e se está em estágio precoce ou tardio, pois enxerga os pacientes e seus familiares com humanidade 5 .

Quando as alterações orgânicas da doença terminal desafiam o tratamento, e quando os alcances do cuidado não podem mais ser cumpridos ou quando o suporte de vida provavelmente levará a resultados insatisfatórios para os pacientes, a equipe multidisciplinar da UTI deve garantir que os pacientes tenham uma qualidade de morte. A definição de qualidade de morte engloba, além das qualidades intrínsecas, as externas, de conforto físico, autonomia, significado, preparação e conexão interpessoal. 0 respeito deve ser promovido, desde a atenção aos significados de qualidade de morte até o evitar danos e conflitos, sendo condições de responsabilidade dos profissionais da saúde cuidar dos pacientes ao final da vida $^{6,7}$.

Atualmente, a presença dos serviços de cuidado paliativo é um elemento adotado como necessário pela Comission on Cancer do American College of Surgeons. Os cuidados paliativos na UTI dão suporte aos pacientes e familiares, podendo proporcionar um ambiente mais confortável e melhor conscientização a respeito da terminalidade da vida, como na extubação paliativa ${ }^{8}$.

A extubação paliativa é descrita como a retirada do tubo traqueal e da ventilação mecânica (VM) quando opções terapêuticas curativas acabaram e essa terapia já não é considerada a melhor para o paciente, para o qual espera-se que a morte ocorra em um curto espaço de tempo. Para a retirada da VM deve-se considerar o quadro clínico do paciente e se o mesmo irá se beneficiar com a extubação, como no caso de ocorrer maior conforto a partir de terapias com esta função, auxílio da equipe multidisciplinar, e também para ocorrer uma melhor comunicação entre paciente e familiar, nessa etapa ${ }^{9}$.

0 processo de comunicação na extubação paliativa envolve percepção, compreensão e transmissão de mensagens na interação entre pacientes, familiares e profissionais da equipe multidisciplinar. Para que possa ser oferecido um serviço de qualidade, a comunicação e tomada de decisões deve ser compartilhada pela equipe multidisciplinar com os familiares, se tornando uma ação essencial para a satisfação familiar. Entre os assuntos abordados deve-se relatar o que esperar durante o processo de extubação e como será o processo de controle dos sintomas, permitindo a presença familiar no momento da morte ${ }^{10}$. 
$\mathrm{Na}$ extubação paliativa, é fundamental o trabalho multiprofissional, sendo medicina, enfermagem e fisioterapia as áreas mais intimamente envolvidas nesse processo. Destaca-se o papel do fisioterapeuta na atuação direta frente alguma das etapas da retirada da VM, principalmente quando ocorre redução dos parâmetros do ventilador, alteração na modalidade ventilatória e extubação do paciente. Mesmo que a extubação paliativa esteja associada à melhora da satisfação e à diminuição da depressão familiar, ainda existem dificuldades para implementar esse procedimento na UTI10,11.

São levantadas discussões sobre o que é melhor para o paciente e familiar, se deve-se continuar com as terapias avançadas convencionais, subestimando o conforto e impondo-lhe sofrimento. As condutas diárias devem ser conduzidas de forma que repense o conforto e o bem-estar do paciente, levando a uma maior qualidade de morte. Por esta razão, colocar em pauta a extubação paliativa é uma forma de reconsiderar que o cuidado paliativo não se apresenta em suas condições ideais, pois presume que não exista diagnóstico precoce para iniciar os cuidados, as condutas de final de vida e a decisão compartilhada com o paciente e familiares para uma melhor aceitação na inevitabilidade da morte.

A manutenção artificial de vida na UTI, como a ventilação mecânica, em muitos casos em que o doente já não apresenta mais qualquer possibilidade de cura, transforma-se em uma extensão prolongada e difícil do processo de morte, para o qual propõe tratamentos inconsistentes e dolorosos para que sobrevivam mediante um sofrimento desnecessário ${ }^{12,13}$. Assim, esse estudo tem como objetivo apresentar revisão acerca do processo da extubação paliativa, assim como seus benefícios na qualidade de morte.

\section{MÉTODO}

Trata-se de uma revisão de literatura integrativa, onde documentos científicos foram obtidos nas plataformas de dados PubMed (US National Library of Medicine National Institutes of Health), Embase (Excerptar Medical Database), Cochrane (The Cochrane Data base of Systematic Reviews) e Scientific Electronic Library Online (SciELO).

A busca nos bancos de dados foi realizada utilizando as terminologias cadastradas nos Descritores em Ciências da Saúde criados pela Biblioteca Virtual em Saúde, desenvolvido a partir do Medical Subject Headings da U.S. National Library of Medicine, que permite o uso da terminologia comum em português, inglês e espanhol.

Os descritores utilizados para a busca foram D060666 Extubação (Airway Extubation) D010166 Cuidados Paliativos (Palliative Care), D003142 Comunicação (Communication), intercalados por meio do operador booleano "AND".

Para a seleção dos artigos realizou-se a leitura dos títulos e resumos, considerando os seguintes critérios: para inclusão foram analisados estudos publicados de 2012 a meados de 2017, que de forma contínua apresentaram a extubação paliativa no processo de qualidade de morte, e aqueles que contemplaram o cuidado paliativo em unidade de terapia intensiva. Documentos cujo acesso se encontrava restrito ao resumo ou ao título, assim como aqueles que não continham as informações necessárias para que os objetivos do presente estudo viessem a serem atingidos, foram excluídos.

Documentos relacionados aos cuidados paliativos e extubação paliativa, selecionados de forma aleatória junto a OMS, também foram inclusos, dada a relevância dos mesmos para a elaboração do presente estudo. Os artigos incluídos foram analisados quanto ao ano de publicação e conteúdo da publicação de forma descritiva.

\section{RESULTADOS}

Através da estratégia de busca, por meio do uso intercalado dos descritores científicos citados anteriormente, foram encontrados 84 documentos científicos na plataforma PubMed, 23 estudos na plataforma Embase, 14 deles na plataforma Cochrane e 11 documentos na 
plataforma Scielo. Foram analisados conforme os critérios de inclusão e exclusão (Fluxograma 1).

Considerou-se 19 artigos na sua íntegra, descritos na Tabela 1, dos quais 1 se tratava do Programa Nacional de Controle do Câncer da OMS, que não está inserido. Dos 19 artigos levantados, 33,33\% apresentaram o cuidado paliativo associado a qualidade de morte, 38,88\% apresentaram os cuidados paliativos inseridos na unidade de terapia intensiva, $27,77 \%$ realizaram a extubação paliativa para uma melhor qualidade de morte.

Nos artigos selecionados, observou-se a importância da equipe multidisciplinar bem preparada, assim como uma boa comunicação com a família e paciente, bem como o desfecho de uma extubação paliativa na qualidade de morte. Por sua vez, os estudos mostram duas realidades:

- a dificuldade que os profissionais da saúde têm em aceitar e lidar com a morte de pacientes, assim como o desafio que é passar este momento aos familiares;

- a dificuldade que esses profissionais, principalmente no Brasil, têm em realizar pesquisas com delineamentos metodológicos rigorosos e adequados no referido assunto, o que, consequentemente, reflete no desfecho do estudo.

Fluxograma 1. Estratégias de busca acerca da extubação, de 2012 a 2017. Franca, 2018.
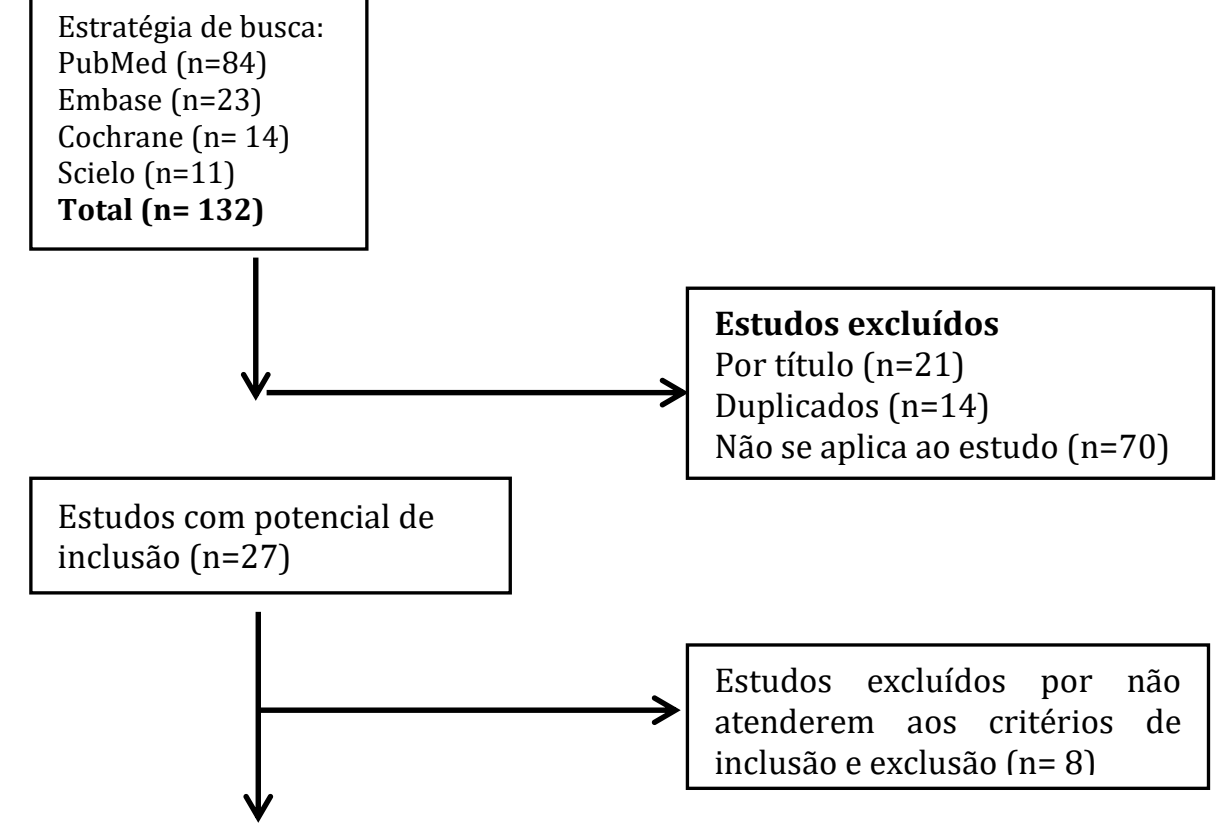

Estudos incluídos na revisão $(n=19)$

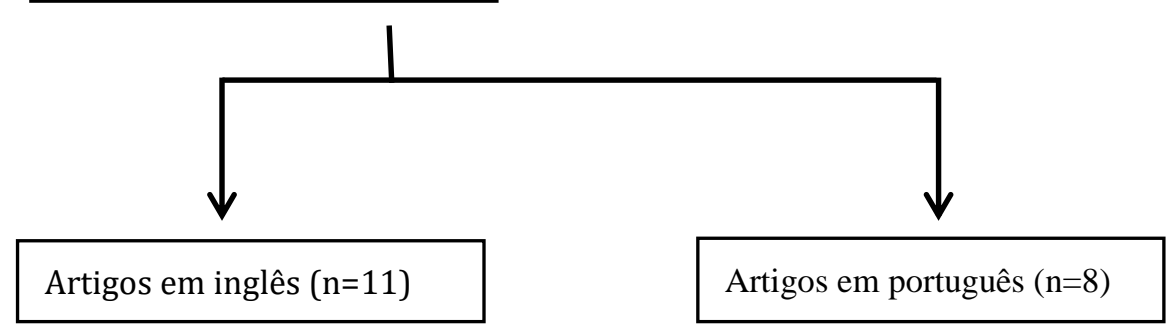


Tabela 1. Artigos acerca da extubação paliativa na qualidade de morte encontrados e considerados, de 2012 a 2017 . Franca, 2018.

\begin{tabular}{|c|c|c|c|c|c|}
\hline Documento Científico & Data & País & Autor(es) & Objetivo(s) & Resultados relevantes \\
\hline $\begin{array}{l}\text { Critically ill cancer } \\
\text { patient in intensive care } \\
\text { unit: issues that arise }{ }^{1}\end{array}$ & 2014 & Grecia & $\begin{array}{l}\text { Kostakou E, Rovina } \\
\text { N, Kyriakopoulou } \\
\text { M, Koulouris NG, } \\
\text { Koutsoukou A. }\end{array}$ & $\begin{array}{l}\text { Observar os cuidados paliativos } \\
\text { quando parece não mais haver } \\
\text { possibilidades de cura. }\end{array}$ & $\begin{array}{l}\text { Estudos adicionais são necessários para avaliar os } \\
\text { resultados médicos a longo prazo e a qualidade de vida } \\
\text { nestes pacientes, a autonomia e a dignidade. }\end{array}$ \\
\hline $\begin{array}{l}\text { O lidar com a morte em } \\
\text { unidade de terapia } \\
\text { intensiva: dificuldades } \\
\text { relatadas } \\
\text { enfermeiros }{ }^{2}\end{array}$ & 2016 & Brasil & $\begin{array}{lr}\text { Lacerda } & \text { CA, } \\
\text { Camboim } & \text { FEF, } \\
\text { Camboim JCA, } \\
\text { Nunes EM, Bezerra } \\
\text { ALD, Sousa MNA } \\
\end{array}$ & $\begin{array}{l}\text { Analisar as dificuldades } \\
\text { enfrentadas por enfermeiros } \\
\text { intensivistas no lidar com a } \\
\text { morte. }\end{array}$ & $\begin{array}{l}\text { Necessita-se de um preparo tanto espiritual como } \\
\text { interpessoal entre os enfermeiros, uma vez que é um } \\
\text { assunto pouco explorado e repleto de dificuldades. }\end{array}$ \\
\hline $\begin{array}{l}\text { Palliative care for the } \\
\text { seriously ill } 4\end{array}$ & 2015 & $\begin{array}{l}\text { Estados } \\
\text { Unidos }\end{array}$ & $\begin{array}{l}\text { Kelley } \\
\text { Morrison RS. }\end{array}$ & $\begin{array}{l}\text { Observar o avanço dos cuidados } \\
\text { paliativos aos doentes graves. }\end{array}$ & $\begin{array}{l}\text { Novos modelos de cuidados paliativos baseados na } \\
\text { comunidade estão surgindo, o conhecimento público e } \\
\text { profissional precisa ser abordado para que o } \\
\text { atendimento possa ser melhorado. }\end{array}$ \\
\hline Cuidados paliativos ${ }^{5}$ & 2016 & Brasil & $\begin{array}{l}\text { Gomes A L Z, } \\
\text { OTheroii M B. }\end{array}$ & $\begin{array}{l}\text { Estudar os conceitos antigos e } \\
\text { atuais, princípios e conceitos } \\
\text { referentes aos } \\
\text { paliativos. }\end{array}$ & $\begin{array}{l}0 \text { cuidado deve ser algo a ser compartilhado, e não } \\
\text { apenas por quem atua na saúde ou em outras áreas do } \\
\text { conhecimento, mas por toda a sociedade. }\end{array}$ \\
\hline $\begin{array}{l}\text { Dying with Dignity in the } \\
\text { Intensive Care Unit } 6\end{array}$ & 2014 & Canada & Cook D, Rocker G. & $\begin{array}{l}\text { A partir de um artigo de revisão, } \\
\text { abordar o conceito de dignidade } \\
\text { para pacientes que morrem em } \\
\text { UTI. }\end{array}$ & $\begin{array}{l}\text { Devemos assegurar que os pacientes sejam auxiliados } \\
\text { durante o processo de morte para que essa ocorra com } \\
\text { dignidade, porém, cria-se conexões que serão } \\
\text { lembradas pelos familiares muito depois da morte. } \\
\text { Solicita-se humanidade de todos participantes da UTI. }\end{array}$ \\
\hline $\begin{array}{l}\text { Respiratory Therapists' } \\
\text { Experiences and } \\
\text { Attitudes Regarding } \\
\text { Terminal Extubations } \\
\text { and End-of-Life Care }{ }^{7} \\
\end{array}$ & 2016 & Georgia & $\begin{array}{l}\text { Grandhige AP, } \\
\text { Timmer M, O'Neill } \\
\text { MJ, Binney ZO, } \\
\text { Quest TE. }\end{array}$ & $\begin{array}{l}\text { Pesquisar terapeutas } \\
\text { respiratórios em dois centros } \\
\text { médicos acadêmicos sobre suas } \\
\text { experiências com extubações } \\
\text { paliativas. }\end{array}$ & $\begin{array}{l}\text { Terapeutas respiratórios raramente são envolvidos em } \\
\text { discussões, apesar do desejo de ser, e experimentam } \\
\text { situações que causam desconforto. Há uma demanda de } \\
\text { treinamento de terapeutas em torno de cuidados para } \\
\text { pacientes terminais. }\end{array}$ \\
\hline $\begin{array}{lr}0 \quad \text { conhecimento de } \\
\text { estratégias } & \text { de } \\
\text { comunicação } & \text { no } \\
\text { atendimento à dimensao } \\
\text { emocional em cuidados } \\
\text { paliativos }{ }^{8}\end{array}$ & 2012 & Brasil & $\begin{array}{l}\text { Araujo MMT, Silva } \\
\text { MJP. }\end{array}$ & $\begin{array}{l}\text { Investigar o conhecimento e a } \\
\text { utilização de estratégias de } \\
\text { comunicação no cuidado da } \\
\text { dimensão emocional do paciente } \\
\text { sob cuidados paliativos. }\end{array}$ & $\begin{array}{l}\text { Conclui-se que há pouco conhecimento e utilização } \\
\text { insatisfatória de estratégias de comunicação, pelos } \\
\text { profissionais de saúde no cuidado à dimensão } \\
\text { emocional de pacientes sob cuidados paliativos. }\end{array}$ \\
\hline $\begin{array}{l}\text { Compassionate } \\
\text { extubation for a peaceful } \\
\text { death in the setting of a } \\
\text { community hospital: a } \\
\text { case-series study }{ }^{9}\end{array}$ & 2015 & China & Kok V C. & $\begin{array}{l}\text { Observar a partir de um estudo } \\
\text { de caso a morte pacífica em um } \\
\text { hospital publico. }\end{array}$ & $\begin{array}{l}\text { O estudo foi realizado de acordo com o protocolo e o } \\
\text { tempo médio de extubação para morte varia } \\
\text { dependendo da doença subjacente, que foi de } 0,3 \text { hora } \\
\text { em pacientes admitidos após parada cardíaca } \\
\text { extrahospitalar e } 97 \text { horas em pacientes com câncer } \\
\text { avançado. }\end{array}$ \\
\hline
\end{tabular}




\begin{tabular}{|c|c|c|c|c|c|}
\hline $\begin{array}{l}\text { Factors Associated With } \\
\text { Family Satisfaction With } \\
\text { End-of-Life Care in the } \\
\text { ICU } 10\end{array}$ & 2015 & $\begin{array}{l}\text { Estados } \\
\text { Unidos }\end{array}$ & $\begin{array}{l}\text { Hinkle LJ, Bosslet } \\
\text { GT , Torke AM }\end{array}$ & $\begin{array}{l}\text { Realizar uma revisão, } \\
\text { sintetizando dados publicados } \\
\text { identificando fatores associados } \\
\text { à satisfação familiar com os } \\
\text { cuidados de fim de vida em } \\
\text { populações adultas criticamente } \\
\text { doentes. }\end{array}$ & $\begin{array}{l}\text { A comunicação de boa qualidade, o suporte para a } \\
\text { tomada de decisões compartilhadas e medidas } \\
\text { específicas de cuidados com os pacientes foram } \\
\text { associados a uma maior satisfação com os cuidados de } \\
\text { fim de vida. }\end{array}$ \\
\hline $\begin{array}{l}\text { Recommendations to } \\
\text { limit life support: a } \\
\text { national survey of } \\
\text { critical care physicians } \\
11\end{array}$ & 2012 & & $\begin{array}{l}\text { Brush DR, Rasinski } \\
\text { KA, Hall JB, } \\
\text { Alexander GC. }\end{array}$ & $\begin{array}{l}\text { Explorar práticas e atitudes } \\
\text { autodenunciadas pelos médicos } \\
\text { referentes aos limites de suporte } \\
\text { de vida. }\end{array}$ & $\begin{array}{l}\text { Os desejos de recomendações dos médicos titulares e o } \\
\text { acordo entre médicos com as decisões prováveis dos } \\
\text { suportes de vida podem ter grande influência na tomada } \\
\text { de decisão, questionando se as recomendações são } \\
\text { seguidas. }\end{array}$ \\
\hline $\begin{array}{l}\text { Protocol in the } \\
\text { management of critical } \\
\text { illness } 12\end{array}$ & 2012 & $\begin{array}{l}\text { Estados } \\
\text { Unidos }\end{array}$ & $\begin{array}{lr}\text { Chang } & \text { SY, } \\
\text { Sevransky } & \text { J, } \\
\text { Martin GS. } & \end{array}$ & $\begin{array}{l}\text { Examinar as vantagens e } \\
\text { desvantagens da protocolização } \\
\text { de cuidados, e mostrar que essa } \\
\text { implementação beneficia os } \\
\text { pacientes. }\end{array}$ & $\begin{array}{l}\text { As vantagens dos protocolos podem ser maximizadas } \\
\text { pelo seu cuidadoso desenvolvimento e implementação, } \\
\text { pela identificação adequada de populações de pacientes } \\
\text { adequadas e pela incorporação de componentes } \\
\text { educacionais. }\end{array}$ \\
\hline $\begin{array}{l}\text { Cuidados paliativos: a } \\
\text { comunicação como } \\
\text { estratégia de cuidado } \\
\text { para o paciente em fase } \\
\text { terminal } 13\end{array}$ & 2013 & Brasil & $\begin{array}{l}\text { Andrade C G } \\
\text { Costa S F G } \\
\text { Lopes M E L }\end{array}$ & $\begin{array}{l}\text { Averiguar como enfermeiros } \\
\text { utilizam a comunicação, } \\
\text { no âmbito dos cuidados } \\
\text { paliativos, ao assistir } \\
\text { o paciente em fase terminal. }\end{array}$ & $\begin{array}{l}\text { Conclui-se que a comunicação se configura como um } \\
\text { elemento eficaz do cuidado com o paciente em fase } \\
\text { terminal e é de suma importância para a promoção dos } \\
\text { cuidados paliativos. Por meio dos depoimentos de } \\
\text { enfermeiros participantes do estudo, observa-se que } \\
\text { estes consideram o relacionamento interpessoal com o } \\
\text { paciente em fase terminal e com seus familiares como } \\
\text { uma ferramenta imprescindível para a promoção dos } \\
\text { cuidados paliativos nessa etapa da vida, haja vista que } \\
\text { lhes permite esclarecer dúvidas, através de uma } \\
\text { linguagem verbal e não verbal que seja simples e } \\
\text { acessível, de forma que os pacientes exteriorizem suas } \\
\text { angústias e seus medos. Outro ponto que merece } \\
\text { destaque diz respeito ao reconhecimento, por alguns } \\
\text { enfermeiros envolvidos na pesquisa, quanto à } \\
\text { participação da família no processo de cuidar do } \\
\text { paciente em fase terminal. }\end{array}$ \\
\hline $\begin{array}{l}\text { Limitação de Suporte } \\
\text { Avançado de Vida em } \\
\text { pacientes admitidos em } \\
\text { unidade de terapia } \\
\text { intensiva com cuidados } \\
\text { paliativos integrados }{ }^{14}\end{array}$ & 2016 & Brasil & $\begin{array}{lrl}\text { Mazutti S } & \text { R } & \text { G, } \\
\text { Nascimento } & A & F, \\
\text { Fumis R RL } & & \end{array}$ & $\begin{array}{l}\text { Estimar a incidência de } \\
\text { limitação de Suporte Avançado } \\
\text { de Vida em pacientes graves } \\
\text { internados em unidade de } \\
\text { terapia intensiva com cuidados } \\
\text { paliativos integrados. }\end{array}$ & $\begin{array}{l}\text { Foi relevante a contribuição dos cuidados paliativos } \\
\text { integrados à unidade de terapia intensiva para a prática } \\
\text { da ortotanásia, ou seja, o não prolongamento da vida de } \\
\text { pacientes críticos terminais por meios artificiais. }\end{array}$ \\
\hline
\end{tabular}


REFACS (online) Abr/Jun 2020; 8(2)

\begin{tabular}{|c|c|c|c|c|}
\hline $\begin{array}{l}\text { Qual a importância dos } \\
\text { Cuidados Paliativos nos } \\
\text { Cuidados } \\
\text { intensivos? }{ }^{15}\end{array}$ & 2015 & Brasil & Catalão D F G. & $\begin{array}{l}\text { Rever a literatura sobre a } \\
\text { importância da aplicação dos } \\
\text { Cuidados Paliativos nas } \\
\text { Unidades de Cuidados } \\
\text { intensivos e seu impacto sobre o } \\
\text { doente, família e equipe médica. }\end{array}$ \\
\hline $\begin{array}{l}\text { Managing end-of-life } \\
\text { decision making in } \\
\text { intensive care medicine } \\
- \text { a perspective from } \\
\text { Charite Hospital } 16\end{array}$ & 2012 & $\begin{array}{l}\text { Alemanh } \\
\text { a }\end{array}$ & $\begin{array}{l}\text { Graw JA, Spies CD, } \\
\text { Wernecke KD, } \\
\text { Braun JP }\end{array}$ & $\begin{array}{l}\text { Observar a tomada de decisões } \\
\text { do fim de vida nos cuidados } \\
\text { intensivos de um hospital de } \\
\text { Charite, na Alemanha }\end{array}$ \\
\hline $\begin{array}{l}\text { Pediatric Critical Care } \\
\text { Transport as a Conduit } \\
\text { to } \\
\text { Terminal Extubation at } \\
\text { Home: A Case Series }{ }^{17}\end{array}$ & 2017 & $\begin{array}{l}\text { Estados } \\
\text { Unidos }\end{array}$ & $\begin{array}{l}\text { Noje C, Bernier M, } \\
\text { Costabile P M, } \\
\text { Klein } \mathrm{B} \quad \mathrm{L} \\
\text {.Kudchadkar S R. }\end{array}$ & $\begin{array}{l}\text { Apresentar a experiência do } \\
\text { centro médico com três } \\
\text { transportes de cuidados } \\
\text { intensivos paliativos da UTIP } \\
\text { para a extubação terminal. }\end{array}$ \\
\hline $\begin{array}{l}\text { Análise descritiva dos } \\
\text { pacientes submetidos à } \\
\text { extubação paliativa }{ }^{18}\end{array}$ & 2015 & Brasil & Rebelatto G. & $\begin{array}{l}\text { Avaliar o perfil clínico- } \\
\text { demográfico dos pacientes } \\
\text { extubados paliativamente na } \\
\text { UTI do Hospital Universitário da } \\
\text { Universidade Federal de Santa } \\
\text { Catarina (HU/UFSC). }\end{array}$ \\
\hline $\begin{array}{l}\text { Factors Associated with } \\
\text { Palliative Withdrawal } \\
\text { of Mechanical } \\
\text { Ventilation and Time to } \\
\text { Death after Withdrawa }{ }^{19}\end{array}$ & 2013 & $\begin{array}{l}\text { Estados } \\
\text { Unidos }\end{array}$ & $\begin{array}{l}\text { Huynh T N, Walling } \\
\text { A M, Le T X, } \\
\text { Kleerup E C, Liu H, } \\
\text { Wenger N S }\end{array}$ & $\begin{array}{l}0 \text { objetivo do estudo foi } \\
\text { identificar fatores associados à } \\
\text { retirada paliativa de ventilação } \\
\text { mecânica e o tempo até a morte } \\
\text { após a extubação. }\end{array}$ \\
\hline
\end{tabular}

A utilização de Cuidados Paliativos nas Unidades de Cuidados Intensivos aumenta a satisfação das famílias, reduz o tempo de internamento nestas unidades e a utilização de medidas fúteis, levando à retirada mais precoce das mesmas, com redução dos custos hospitalares sem alteração da mortalidade.

$\mathrm{Na}$ UTI, os procedimentos de fim de vida foram realizados individualmente. Porém, eles seguem um padrão de tomada de decisão compartilhada com cuidados na substituição do paciente.

A promulgação de uma lei sobre diretrizes antecipadas não afetou o processo de tomada de decisão em situações de fim de vida e nem afetou os hábitos de planejamento de cuidados avançados da população. No entanto, isso levou a uma maior documentação associada a situações de fim de vida na UTI.

Embora uma prática relativamente pouco frequente em cuidados críticos pediátricos, o transporte para casa para a extubação terminal representa uma alternativa viável para as famílias que procuram cuidados de fim de vida fora do hospital para seus filhos criticamente dependentes da tecnologia.

Os pacientes que foram extubados eram mais idosos, acometidos especialmente por doenças neurológicas e o tempo médio entre a extubação e o óbito foi de 2,5 dias.

A retirada paliativa da ventilação mecânica foi realizada em apenas metade dos pacientes com ventilação mecânica falhando. Uma vez que o serviço clínico, em vez dos parâmetros fisiológicos, estão associados à retirada, as intervenções direcionadas podem melhorar as decisões de retirada. 


\section{DISCUSSÃO}

A extubação paliativa é uma forma de conforto para uma melhor qualidade de morte que vem ganhando espaço em pacientes terminais internados em UTI. Um dos estudos revelou ${ }^{13}$ que muitas vezes é visto que o desejo do paciente e dos familiares é de poder estar os últimos momentos em casa, cercado de familiares e amigos. Este cenário mostra que o que os pacientes desejam é nada mais que conforto, companhia, bom humor e uma boa conversa. Outro estudo relata que, em alguns casos, é possível realizar esse pedido. Porém, essa não é a realidade do Brasil, sendo muitas vezes difícil satisfazer essa vontade. São realizadas então medidas para estimular a satisfação dos pacientes e simular o ambiente familiar na UTI, e devem ser encorajadas. ${ }^{14}$

Um estudo ${ }^{16}$ mostrou que a extubação permite que o paciente fique livre desse procedimento indesejado, possibilitando maior comunicação entre paciente e família, abreviando o processo da morte quando a cura já não é uma opção e muitas vezes causa dor e sofrimento. Pode ser considerado um aspecto negativo deste método a família interpretar a respiração ruidosa das vias aéreas como um desconforto do paciente. Porém, em outro estudo $^{10}$, os familiares relataram maior satisfação quando foram permitidos permanecer por tempo mais prolongado durante as conferências de tomadas de decisões, e durante o processo de extubação. Por esta razão o acompanhamento da equipe se faz necessário, visto que a administração de sedativos, técnicas para diminuir a dor e desconforto, podem ser utilizadas $^{10,15,16}$.

Um estudo ${ }^{17}$ foi realizado no Hospital Johns Hopkins, com três pacientes pediátricos que tinham como vontade própria e de sua família passar os últimos momentos em casa. A extubação paliativa foi realizada por um terapeuta respiratório paliativista, que relatou que os três pacientes morreram pacificamente logo após a extubação, porém, estavam assegurados de conforto e cercados de sua família, proporcionando um cuidado ao final da vida com compaixão, passivo e sensível. Foi identificada a importância do pré-planejamento, comunicação efetiva com a família e membros da equipe, conseguidos a partir de cinco fases de extubação fora da UTI: introdução de retirada, preparação pré-transferência, extubação, cuidados pós-extubação e cuidado com a qualidade de morte ${ }^{17}$.

Outro estudo, realizado em Florianópolis ${ }^{18}$, relatou que $53,8 \%$ dos pacientes que morreram receberam abordagem paliativista. Entretanto, a decisão de extubação paliativa foi de apenas 3,35\% do número total de óbitos da unidade. A maioria dos profissionais que trabalham em UTI relaciona o bem-estar do paciente dispneico ao fornecimento de oxigênio e à VM. A falta de estudos por parte desses profissionais faz com que relacionem a retirada da VM ao conceito de eutanásia. A baixa extubação paliativa encontrada no estudo confirmam essas afirmações. 0 estudo constatou também que o tempo de morte após a extubação paliativa variou de minutos a dias, chegando ao máximo de 6 a 9 dias ${ }^{18}$.

Ao avaliar a decisão de extubação paliativa, percebe-se a preocupação de que esse ato traga estresse aos familiares dos pacientes, porém, estudos revisados recentemente apontam o inverso, demonstrando que os membros das famílias de pacientes que morreram após o extubação paliativa na UTI relataram menos sintomas de depressão. Infelizmente, mesmo com a comprovação de que a extubação paliativa traz conforto ao paciente e seus familiares, fatores culturais e religiosos estão relacionados à distanásia ${ }^{9,19}$.

\section{CONCLUSÃo}

Conclui-se que a extubação paliativa, quando empregada corretamente, com o auxílio de toda equipe multidisciplinar, em comum acordo com os familiares e realizando o desejo do paciente, é de extrema importância, oferecendo uma melhor qualidade de morte a estas pessoas quando seu tratamento já não é mais possível, e prolongaria portanto o seu sofrimento e de seus familiares. 
A presente revisão tem como limitações a escassa quantidade de estudos relacionados à extubação paliativa, tendo em vista que esse procedimento pode ser entedido de forma errada pelos familiares e muitas vezes por outros profissionais da saúde, além da heterogeneidade dos estudos e as amostras pequenas e pouco controladas.

\section{REFERÊNCIAS}

1. Kostakou E, Rovina N, Kyriakopoulou M, Koulouris NG, Koutsoukou A. Critically ill cancer patient in intensive care unit: issues that arise. J Crit Care [Internet]. 2014 [citado em $06 \mathrm{fev}$ 2020]; 29(5):817-22. Disponível

em: https://www.sciencedirect.com/science/article/pii/S0883944114001452.

DOI: https://doi.org/10.1016/j.jcrc.2014.04.007

2. Lacerda CA, Camboim FEF, Camboim JCA, Nunes EM, Bezerra ALD, Sousa MNA. O lidar com a morte em unidade de terapia intensiva: dificuldades relatadas por enfermeiros. C\&D Rev Elet da FAINOR [Internet]. 2016 [citado em 06 fev 2020]; 9(2):173-86. Disponível em: http://srv02.fainor.com.br/revista/index.php/memorias/article/view/559

3. World Health Organization. National cancer control programmes: policies and managerial guidelines. Genève: WHO; 2012. Disponível em: https://www.who.int/cancer/publications/nccp2002/en/

4. Kelley AS, Morrison RS. Palliative care for the seriously ill. N Engl J Med. [Internet] 2015 [citado em 06 fev 2020]; 373(8):747-55 Disponível em: https://www.nejm.org/doi/full/10.1056/NEJMra1404684

5. Gomes ALZ, Othero MB. Cuidados paliativos. Estud Av. [Internet]. 2016 [citado em 06 fev 2020]; 30(88): Disponível em: http://www.scielo.br/pdf/ea/v30n88/0103-4014-ea-30-880155.pdf. DOI: DOI: 10.1590/S0103-40142016.30880011

6. Cook D, Rocker G. Dying with dignity in the Intensive Care Unit. N Engl J Med. [Internet]. 2014 [citado em 06 fev 2020]; 370:2506-14. Disponível em: https://www.nejm.org/doi/full/10.1056/NEJMra1208795

7. Grandhige AP, Timmer M, O'Neill MJ, Binney ZO, Quest TE. Respiratory therapists' experiences and attitudes regarding terminal extubations and end-of-life care. Respir Care [Internet]. 2016 [citado em 06 fev 2020]; 61(7):891-6. Disponível em: https://www.ncbi.nlm.nih.gov/pubmed/27274094. DOI: doi: 10.4187/respcare.04168

8. Araujo MMT, Silva MJP. O conhecimento de estratégias de comunicação no atendimento à dimensão emocional em cuidados paliativos. Texto \& Contexto Enferm. [Internet]. 2012 [citado em 06 fev 2020]; 21(1): 121-9. Disponível em: http://www.scielo.br/pdf/tce/v21n1/a14v21n1.pdf

9. Kok VC. Compassionate extubation for a peaceful death in the setting of a community hospital: a case-series study. Clin Interv Aging [Internet]. 2015 [citado em 06 fev 2020]; 10:67985. Disponível em: https://www.ncbi.nlm.nih.gov/pmc/articles/PMC4396346/. DOI: 10.2147/CIA.S82760

10. Hinkle LJ, Bosslet GT, Torke AM. Factors associated with family satisfaction with end-of-life care in the ICU. Chest [Internet]. 2015 [citado em $06 \mathrm{fev} 2020$ ]; 147(1):82-93. Disponível em: https://www.ncbi.nlm.nih.gov/pubmed/25103451. DOI: 10.1378/chest

11. Brush DR, Rasinski KA, Hall JB, Alexander GC. Recommendations to limit life support: a national survey of critical care physicians. Am J Respir Crit Care Med. [Internet]. 2012 [citado em 06 fev 2020]; 186(7):633-9. Disponível em: https://www.ncbi.nlm.nih.gov/pubmed/22837382. DOI: 10.1164/rccm.201202-03540C

12. Chang SY, Sevransky J, Martin GS. Protocol in the management of critical illness. Crit Care [Internet]. 2012 [citado em 06 fev 2020]; 16(2):306. Disponível em: https://www.ncbi.nlm.nih.gov/pmc/articles/PMC3584719/ 
13. Andrade CG, Costa SFG, Lopes MEL. Cuidados paliativos: a comunicação como estratégia de cuidado para o paciente em fase terminal. Ciên Saúde Colet [Internet]. 2013 [citado em 06 fev 2020]; 18(9):2523-30. Disponível em: http://www.scielo.br/pdf/csc/v18n9/v18n9a06.pdf 14. Mazutti SRG, Nascimento AF, Fumis RRL. Limitação de Suporte Avançado de Vida em pacientes admitidos em unidade de terapia intensiva com cuidados paliativos integrados. Rev Bras Ter Intensiva [Internet]. 2016 [citado em 06 fev 2020]; 28(3):294-300. Disponível em: http://www.scielo.br/pdf/rbti/v28n3/0103-507X-rbti-20160042.pdf. DOI: 10.5935/0103507X.20160042

15. Catalão DFG. Qual a importância dos cuidados paliativos nos cuidados intensivos?[Internet]. [dissertação]. Porto, Portugal: Universidade do Porto; 2015. [acesso em 31 out 2017]. Disponivel em: https://sigarra.up.pt/fcnaup/pt/pub_geral.pub_view?pi_pub_base_id=37489 16. Graw JA, Spies CD, Wernecke K-D, Braun J-P. Managing end-of life decision making in intensive care medicine - a perspective from Charité Hospital, Germany. PLos ONE [Internet]. 2012 [citado em 06 fev 2020]. 7(10):e46446. Disponível em: https://journals.plos.org/plosone/article?id=10.1371/journal.pone.0046446. DOI: https://doi.org/10.1371/journal.pone.0046446

17. Noje C, Bernier ML, Costabile PM, Klein BL, Kudchadkar SR. Pediatric critical care transport as a conduit to terminal extubation at home: a case series. Pediatr Crit Care Med. [Internet]. 2017 [citado em 06 fev 2020]; 18(1):e4-e8. Disponível em: https://www.ncbi.nlm.nih.gov/pubmed/27801708. DOI: 10.1097/PCC.0000000000000997

18. Rebelatto G. Análise descritiva dos pacientes submetidos à extubação paliativa [Internet]. [dissertação]. Florianópolis: Universidade de Santa Catarina; 2015 [citado em 31 out 2017]. Disponivel em: https://repositorio.ufsc.br/handle/123456789/169536

19. Huynh TN, Walling AM, Le TX, Kleerup EC, Liu H, Wenger NS. Factors associated with palliative withdrawal of mechanical ventilation and time to death after withdrawal. J Palliat Med. [Internet]. 2013 [citado em 06 fev 2020]; 16(11):1368-74. Disponível em: https://www.ncbi.nlm.nih.gov/pubmed/24083651. DOI: 10.1089/jpm.2013.0142

\section{CONTRIBUIÇÕES}

Mariana Fernandes Peixoto contribuiu na concepção, delineamento, analise e interpretação de dados e redação. Beatriz Paschoini de Andrade Silva e Fabio Bruno Silva Nascimento participaram no delineamento. Daniela Santana Polati da Silveira atuou na análise e interpretação de dados e revisão.

\section{Como citar este artigo (Vancouver)}

Peixoto MF, Nascimento FBS, Silva BPA, Silveira DSP. Os benefícios da extubação paliativa na qualidade de morte. REFACS [Internet]. 2020 [citado em inserir dia, mês e ano de acesso]; 8(2):307-316. Disponível em: inserir link de acesso. DOI: inserir link do DOI.

\section{Como citar este artigo (ABNT)}

PEIXOTO, M. F.; NASCIMENTO, F. B. S.; SILVA, B. P. A.; SILVEIRA, D. S. P. Os benefícios da extubação paliativa na qualidade de morte. REFACS, Uberaba, MG, v. 8, n. 2, p. 307-316, 2020. Disponível em: inserir link de acesso. Acesso em: inserir dia, mês e ano de acesso. DOI: inserir link do DOI.

\section{Como citar este artigo (APA)}

Peixoto, M.F., Nascimento, F.B.S., Silva, B.P.A., \& Silveira, D.S.P. (2020). Os benefícios da extubação paliativa na qualidade de morte. REFACS, 8(2), 307-316. Recuperado em: inserir dia, mês e ano de acesso de inserir link de acesso. DOI: inserir link do DOI. 